\title{
Dual-energy computed tomography of the neck-optimizing tube current settings and radiation dose using a 3D-printed patient phantom
}

\author{
Torsten Diekhoff ${ }^{1}$, Michael Scheel ${ }^{1}$, Wiebke Kress ${ }^{1}$, Bernd Hamm ${ }^{1}$, Paul Jahnke ${ }^{1,2}$ \\ ${ }^{1}$ Department of Radiology, Charité - Universitätsmedizin Berlin, Campus Mitte, Humboldt-Universität zu Berlin, Freie Universität Berlin, Berlin, \\ Germany; ${ }^{2}$ Berlin Institute of Health (BIH), Anna-Louisa-Karsch-Str. 2, 10178 Berlin, Germany
}

Correspondence to: Dr. Torsten Diekhoff, MD. Charité - Universitätsmedizin Berlin, Department of Radiology (CCM), Charitéplatz 1, 10117 Berlin, Germany. Email: torsten.diekhoff@charite.de.

Background: Dual-energy computed tomography (DECT) is increasingly used in studies and clinical practice. However, the best protocol is controversially discussed and whether it exhibits more radiation exposure compared to conventional protocols. Thus, the purpose of the study was to determine optimal tube current settings for DECT in a 3D-printed anthropomorphic phantom of the neck.

Methods: A 3D-printed iodinated ink based phantom of a contrast enhanced CT of the neck was imaged. Six dual-energy multi-detector computed tomography scans were performed with six different tube currents (80 kVp: 30-400 mAs; $135 \mathrm{kVp}$ : 5-160 mAs). 120 virtual blended images (VBIs) and 66 virtual monochromatic images (VMIs) were reconstructed and 12 regions of interest (bilaterally: common carotid arteries, subcutaneous soft tissue, mandibular bone, sternocleidomastoid muscle, submandibular gland, and mid-image: vertebral body of C2 and pharyngeal space) in six consecutive slices resulting in 96 measurements per scan were performed. Hounsfield units and signal- and contrast-to-noise ratio were compared to singleenergy computed tomography as standard of reference.

Results: VBIs overestimated the Hounsfield units $(\mathrm{P}<0.0001)$. Optimal dual-energy scanning parameters resulted in 120\% (100 kVe: 51.2 vs. 61.7 and 65.2, for signal and contrast-to-noise ratio, respectively; 120 kVe: 60.8 vs. 72.1 vs. 128.3 ) of the radiation exposure with about $80 \%$ of the signal/contrast-to-noise ratio of the corresponding single-energy images. However, optimal weighting of tube currents for both voltages depended on the desired reconstruction.

Conclusions: Dual-energy protocols apply an estimated $120 \%$ of the single-energy radiation exposure and result in approximately $80 \%$ of the image quality. Tube current settings should be adapted to the desired information.

Keywords: Phantoms; imaging; printing; three-dimensional; tomography; X-ray computed; radiation exposure

Submitted Jul 11, 2020. Accepted for publication Oct 20, 2020.

doi: $10.21037 /$ qims-20-854

View this article at: http://dx.doi.org/10.21037/qims-20-854

\section{Introduction}

Dual-energy computed tomography (DECT), also called spectral CT, gained importance during recent years along with the development of different clinical applications. It provides opportunities for advanced material characterization by using the attenuation at two or more energy levels (i.e., tube voltages) to deduce the effective atomic number (effective $Z$ ) of an object $(1,2)$. Especially materials with high effective $Z$ such as iodinated contrast media can be visualized through their difference in attenuation by the photoelectric effect and Compton scattering, which can be expressed by a material-specific 


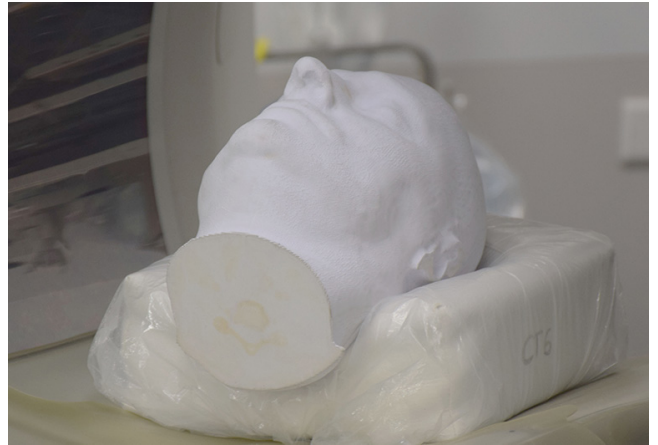

Figure 1 Photography of the phantom.

gradient $(3,4)$.

DECT is therefore interesting for applications with contrast media $(5,6)$, e.g., the reconstruction of virtual non-enhanced images $(7,8)$ or iodine maps $(9,10)$. The quantification of iodine uptake using DECT iodine maps in patients with squamous cell carcinomas showed value for the prediction of recurrence after radiotherapy (11). However, DECT also allows to reconstruct virtual blended images (VBIs) and virtual monochromatic images (VMIs). VBIs are calculated as average of the differently weighted DECT source images (12) and aim to imitate conventional single energy CT (SECT) images. They can be reconstructed at variable energy levels and thereby simulate the whole $\mathrm{X}$-ray spectrum of a conventional CT machine (e.g., 80, 100 or 120 kilovolt peak; $\mathrm{kVp}$ ). VMIs simulate patient imaging with a monochromatic beam instead of an X-ray spectrum as used for single energy CT (e.g., 70 kiloelectronvolt; $\mathrm{keV})$. VMIs with low $\mathrm{keV}$ can increase the image contrast and thus compensate low contrast medium concentrations (13-15). VMIs with high $\mathrm{keV}$ allow for the reduction of metal artefacts, e.g., of dental implants (16-18).

While the diagnostic benefits of DECT are at hand, it is controversial if the application of DECT exhibits more radiation exposure compared to SECT or if DECT can be performed while maintaining or improving the image quality with the same dose as SECT (19-21). To this end, we wanted to extrapolate optimal tube current settings from a variety of combinations that would result in the best ratio of image quality and radiation exposure. For this study, we used a novel anthropomorphic three-dimensional printed phantom based on a clinical CT-scan of the neck and performed different SECT and DECT scans and a variety of DECT post-processing procedures. The phantom allowed for repeated measurements at different energy levels and with varying radiation exposure while imitating the anatomy of a patient. The aim of the study was to deduce optimal tube current settings for a DECT scan of the neck with acceptable image quality and lowest possible radiation exposure compared to clinical standard SECT.

\section{Methods}

\section{Phantom}

A radiopaque $3 \mathrm{D}$ printed neck phantom was used for simulation of patient imaging (see Figure 1). This approach allowed for a large number of repeated exposures and measurements in an anatomically accurate object, which is not possible with conventional phantoms or patient cohorts. The phantom has precise density values that reflect different iodine concentrations and, thus, contrast enhancement. The anatomical correctness takes into account the patients' microstructure and possible geometrical artefacts. The phantom was manufactured from a random clinical contrast enhanced CT scan of a patient. The CT images were printed with aqueous potassium iodide doped ink $(600 \mathrm{mg} / \mathrm{mL})$ on paper using previously published methods to reproduce patient anatomy and attenuation values (22). The printed paper sheets were then assembled to a phantom using paper-based 3D printing, where the paper sheets are stacked, glued and cut to the patient shape (23). The phantom thus consisted of a paper base material, potassium iodide and glue. It covered $22.5 \mathrm{~cm}$ in z-axis and had the same size and contour as the patient. Internal airways (e.g., pharynx) were represented by unprinted paper, which was not removed from this phantom.

\section{Scanning protocol}

A 320 rows detector CT system (Canon Aquilion One Vision edition; Canon Medical Systems) was used for image acquisition. The scanning protocol consisted of a standard scanogram, a set of four clinical single-energy CTs (SECT) that were used as standard of reference for most image quality evaluations and a set of dual-energy CT (DECT) scans to provide the basic datasets for the following reconstructions. The SECT and DECT scans were performed in volume mode using a medium field-of-view without table movement.

\section{SECT scanning protocol}

Four scans with different tube voltages were acquired using clinical standard settings for the neck with enabled automated exposure control [standard deviation $(\mathrm{SD})$ value of 12 and a maximum tube current of $600 \mathrm{~mA}$ ], which 
reflects the standard of clinical care in our institution. The applied tube voltages were $80,100,120$ and $135 \mathrm{kVp}$. Thus, the whole spectrum of the CT machine was used. For a better comparison with the DECT datasets, a volume scan technique with $16 \mathrm{~cm} \mathrm{z}$-axis coverage and a rotation time of $0.5 \mathrm{~s}$ was used instead of a spiral scan. The volume mode utilizes the whole width of the detector ( $16 \mathrm{~cm}, 320$ rows) without table movement, thus, acquiring up to 640 images in one gantry rotation.

\section{DECT scanning protocol}

Six DECT scans were performed with 80 and $135 \mathrm{kVp}$ tube voltage and an ascending order of tube currents $(30,55$, 115, 230 and $400 \mathrm{mAs}$ for $80 \mathrm{kVp}$ and 5, 10, 20, 40, 80 and $160 \mathrm{mAs}$ for $135 \mathrm{kVp}$ ). No tin-filter was applied. The automatic exposure control was disabled to allow a structured thorough analysis of low and high radiation exposure within the limits of the scanning system. The rotation time was $0.5 \mathrm{~s}$.

\section{Image reconstruction}

We used a medium soft tissue kernel without beam hardening compensation (FC13) for the reconstruction of each volume dataset to $0.5 \mathrm{~mm}$ isotropic voxel. All images were reconstructed using iterative reconstruction (AIDR-3D standard).

The images were further processed using the CT console and two software applications: (I) Dual-Energy Image View (Version 6, Canon Medical Systems) and (II) Dual-Energy Raw Data Analysis (Version 6, Canon Medica Systems) for the reconstruction of (I) VBIs and (II) VMIs. Applicationinherent registration algorithms were applied.

All acquired 80 and $135 \mathrm{kVp}$ DECT source datasets were combined with each other for the reconstruction of VBIs. This resulted in a total of 30 source dataset pairs. For VMIs only the pairings recommended by the vendor could be used due to restrictions of the software [6 pairings (tube current at high/low tube voltage): 5/30, 10/55, 20/115, 40/230, $80 / 400$, and $160 / 400 \mathrm{mAs}$. VBIs were reconstructed at 80, 100, 120 and 135 kilo Volt equivalent (kVe) and VMIs with $35,45,55,65,75,85,95,105,115,125$ and $135 \mathrm{keV}$.

\section{Measurements and calculations}

We defined twelve circular regions of interest (ROIs) with $10 \mathrm{~mm}^{2}$ area, each on six consecutive $0.5 \mathrm{~mm}$ axial planes within the phantom (1st left common carotid artery, $2^{\text {nd }}$ right common carotid artery, $3^{\text {rd }}$ left subcutaneous soft tissue, $4^{\text {th }}$ right subcutaneous soft tissue, $5^{\text {th }}$ left mandibular bone, $6^{\text {th }}$ right mandibular bone, $7^{\text {th }}$ left sternocleidomastoid muscle $8^{\text {th }}$ right sternocleidomastoid muscle, $9^{\text {th }}$ left submandibular gland, $10^{\text {th }}$ right submandibular gland, $11^{\text {th }}$ vertebral body of $\mathrm{C} 2,12^{\text {th }}$ pharyngeal space-reflecting different iodine concentrations within the phantom) and one ROI in the air ventral, posterior, left and right of the phantom, respectively. As there was no table movement between the scans, the ROIs could precisely be co-registered using the table position/slice number. Signal-to-noise ratios (SNR) and contrast-to-noise ratios (CNR) were calculated according to Eqs. [1] and [2], respectively.

$$
S N R=\frac{\text { signal }}{\text { noise }}=\frac{\text { mean } H U_{R O I}}{\text { mean } S D_{\text {air }}}
$$

Eq. [1]: calculation of signal-to-noise ratio. SNR: signalto-noise ratio; mean $\mathrm{HU}$ : mean attenuation in Hounsfield units (HU) measured in the organ ROI; mean SD: mean standard deviation measured in all air ROI placed in air outside the phantom.

$$
\begin{aligned}
C N R & =\frac{\text { signal }_{\text {organ }}-\text { signal }_{\text {phantom }}}{\text { noise }} \\
& =\frac{\text { mean } H U_{\text {ROI } 1-11}-\text { mean } H U_{R O I 12}}{\text { mean } S D_{\text {air }}}
\end{aligned}
$$

Eq. [2]: calculation of the contrast-to-noise ratio. CNR: contrast-to-noise ratio; mean $\mathrm{HU}$ : mean attenuation in Hounsfield units (HU); mean SD: mean standard deviation measured in all air ROI placed in air outside the phantom.

\section{Primary endpoints and statistics}

For all measurements the corresponding SECT dataset was used as standard of reference. A repeated measurements ANOVA and Dunnett's multiple comparison test was used for different calculations: (I) to compare $\mathrm{HU}$ of different VBI reconstructions with corresponding SECT datasets (e.g., $100 \mathrm{kVe}$ VBI with $100 \mathrm{kVp}$ SECT), (II) to compare HU of different VMIs with the four SECT datasets. SNR and CNR values of VBI and VMI reconstructions were compared graphically by plotting the respective image quality value (SNR or CNR) against the radiation exposure as measured by the dose length product (DLP). A nonlinear second order regression analysis was performed to project a curve from the different data points. The optimal image settings were defined as the point on the curve being closest to the reference SECT scan, i.e., having the 

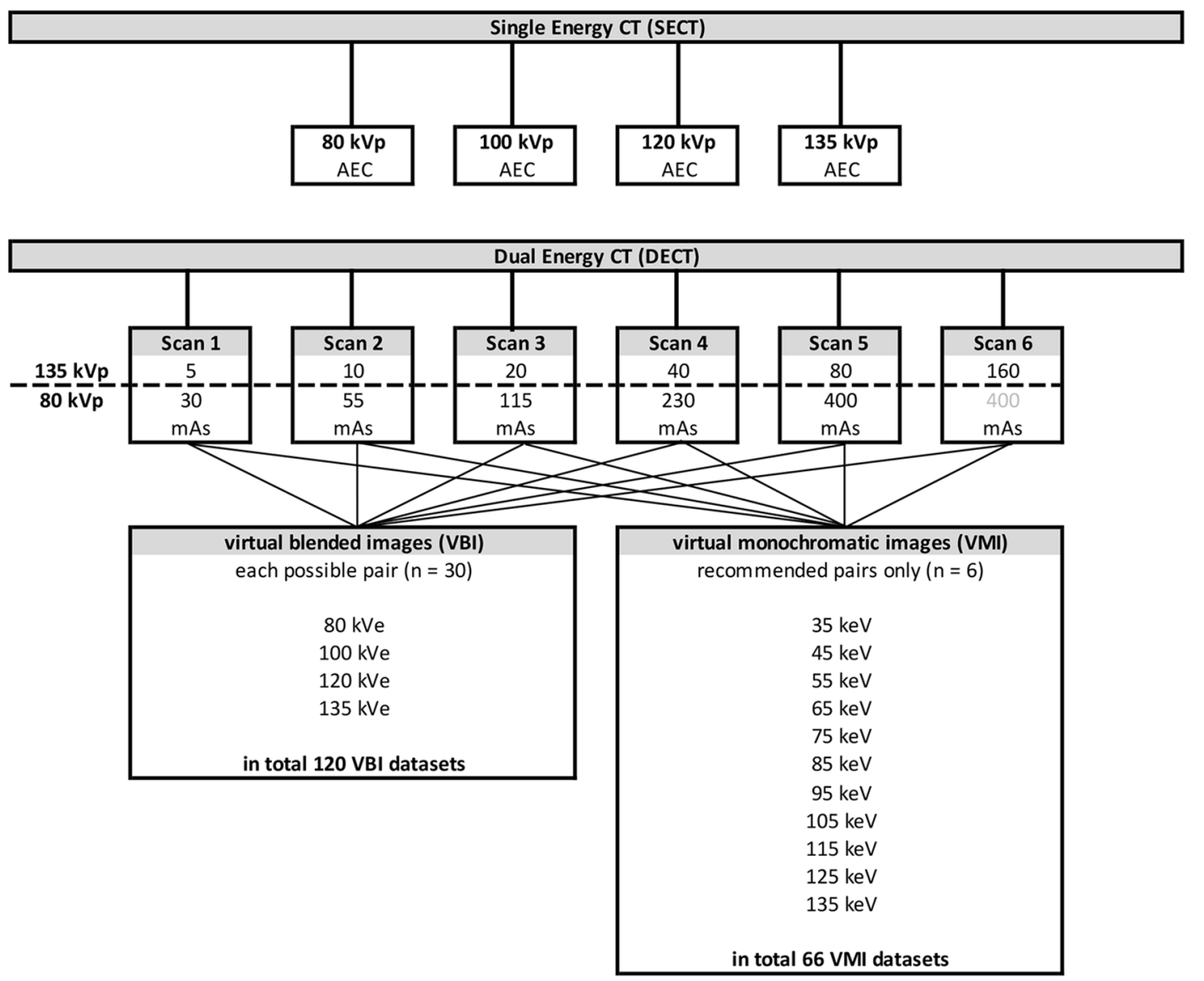

In total 4 SECT and 186 DECT datasets

Figure 2 Flow chart of single energy computed tomography (SECT) and dual energy computed tomography (DECT) scans and image reconstructions. Four SECT images were reconstructed and served as standard of reference. In total 186 DECT images were reconstructed: for virtual blended images (VBIs) all possible pairings of tube current for high and low tube voltage of the six different DECT scans were used $(n=30)$, for virtual monochromatic images only the recommended pairings were used $(n=6)$.

most similar combination of image quality and radiation exposure. For this purpose, the minimum of the following distance Eq. [3] was calculated.

$$
d(x)=\sqrt[2]{(x-A)^{2}+\left(B_{2} x^{2}+B_{1} x+B_{0}-B\right)^{2}}
$$

Eq. [3]: distance $(\mathrm{d}(\mathrm{x}))$ of the single energy dataset (point (A | B)) from the dual energy function with the formula $f(x)=B_{2} x^{2}+B_{1} x+B_{0}$. The graph formula was derived from a non-linear second order regression analysis of signal (or contrast) to noise ratio (y-axis) and total radiation exposure [DLP] (x-axis).

\section{Results}

The following datasets were included in the analysis: 4 SECT, 120 VBI and 66 VMI datasets (see Figure 2). For each dataset, 96 ROI measurements were performed resulting in a total of 18,240 measurements. Exemplary images are provided in Figure 3.

\section{Hounsfield units}

The mean measured HU of the reference SECT datasets, reconstructed VBI and VMIs are shown in Figure 4. We observed overexposure in the $135 \mathrm{kVp} / 160 \mathrm{mAs}$ DECT 


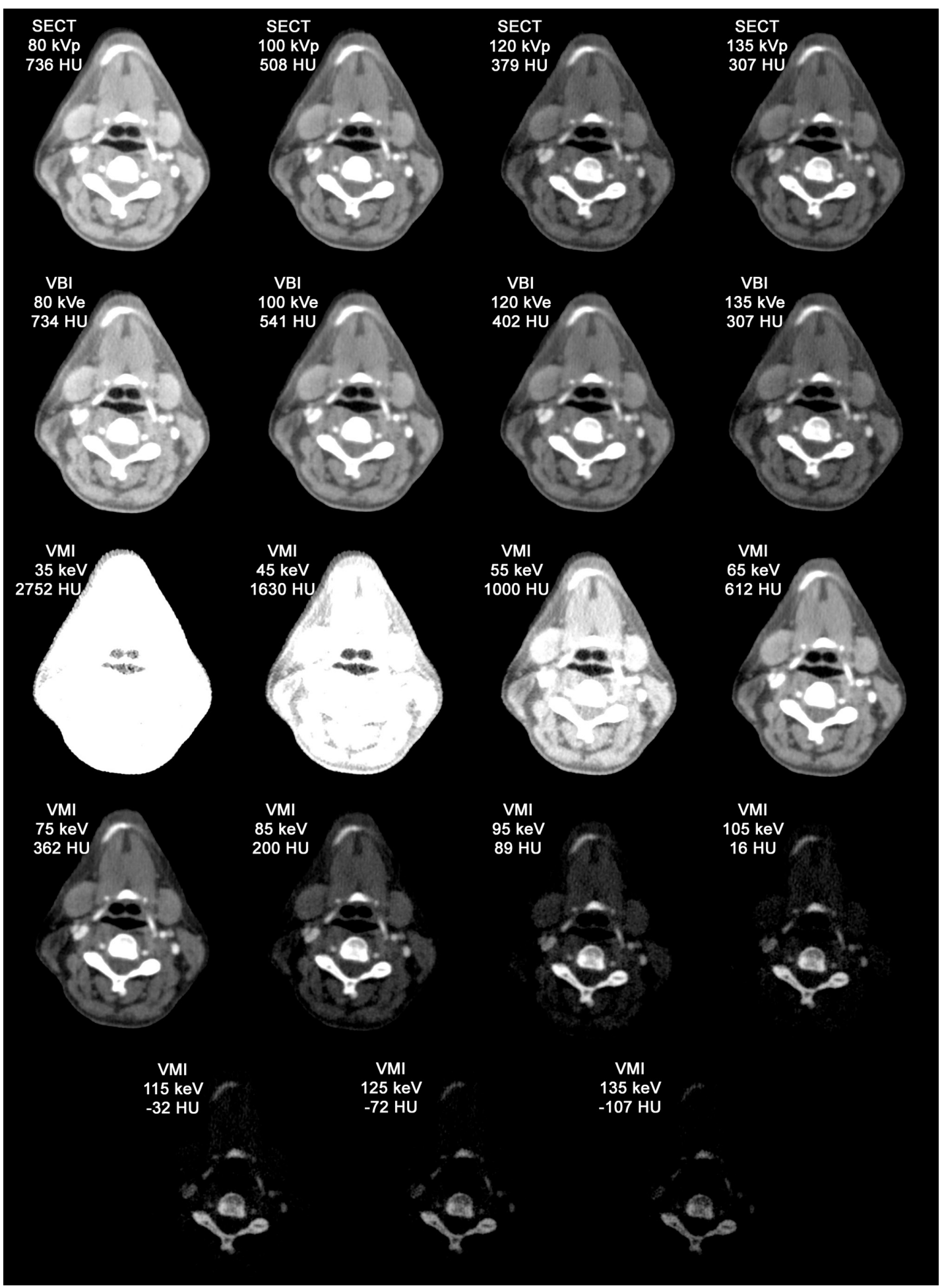

Figure 3 Example images of single energy computed tomography (SECT) scans and dual energy computed tomography (DECT) reconstructions. The DECT reconstructions were derived from the 20/115 mAs (high/low energy) dataset. Images are presented with window level 240 and window width 730. The given HU value represents the mean of all ROI measurements in from this scan. VBI, virtual blended images; VMI, virtual monochromatic images. 

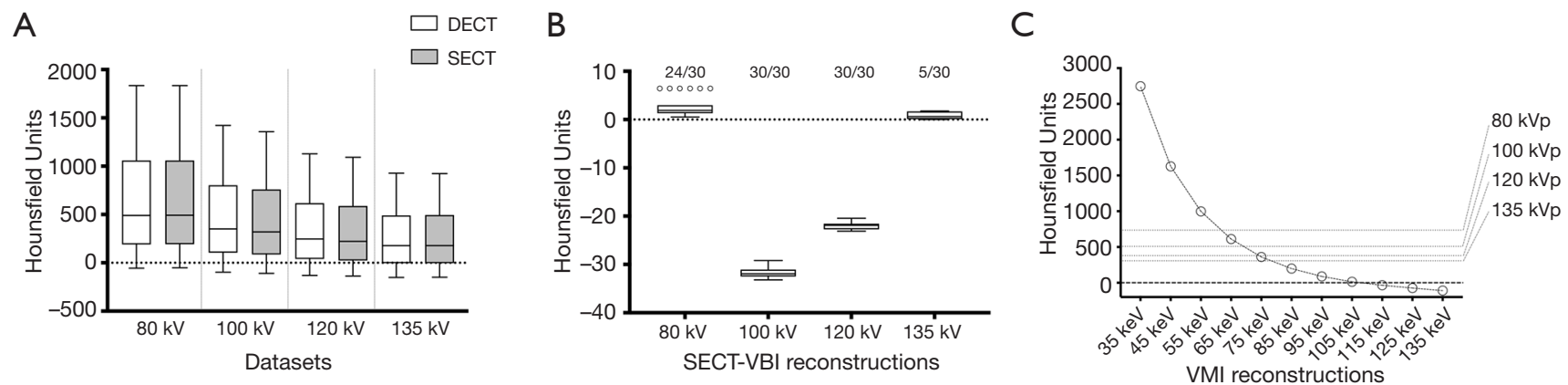

Figure 4 Hounsfield units (HU) of virtual blended images (VBI) and virtual monochromatic images (VMI) compared to single energy computed tomography (SECT). (A) Virtual blended images in 80, 100, 120 and $135 \mathrm{kVe}$ compared to the corresponding SECT images. (B) Differences in HU between SECT and VBI. Numbers of statistically significant deviations from Dunnett's multiple comparison test are shown on top (see also Appendix 1A-D). (C) Comparison of HU between VMI and SECT. The horizontal lines indicate the corresponding values of the SECT datasets. There was no difference between the $75 \mathrm{keV}$ VMI and $120 \mathrm{kVp}$ SECT. All plotted values are the mean values of all ROI measurements of the respective reconstruction.

source dataset that was transferred to related reconstructions.

\section{VBIs}

The mean \pm SD difference of the HU measured in the corresponding SECT images and VBI (HUSECT HUVBI) was $2.63 \pm 2.1 \mathrm{HU}$ for $80 \mathrm{kVe}$ and did not reach significance for pairings based on the $400 \mathrm{mAs}$ dual-energy source dataset, $-31.68 \pm 1.18 \mathrm{HU}$ for $100 \mathrm{kVe}(\mathrm{P}<0.0001$ for all pairings), $-22.03 \pm 0.75 \mathrm{HU}$ for $120 \mathrm{kVe}(\mathrm{P}<0.0001$ for all pairings) and $0.79 \pm 0.75 \mathrm{HU}$ for $135 \mathrm{kVe}$ (only significant for pairings based on the $160 \mathrm{mAs}$ dataset). The detailed results of the multiple comparison analysis can be found in Appendix 1A-D, a graphical version is presented in Figure $4 A$ and $B$.

\section{VMIs}

There was no statistical difference of HU between the $120 \mathrm{kV}$ SECT and the $75 \mathrm{keV}$ VMI images (mean difference $16.8 \pm 19.0 \mathrm{HU}$ ). All other dual-energy pairings showed highly significant differences to all SECT images $(\mathrm{P}<0.001)$. A graphical comparison of the measured $\mathrm{HU}$ in VMI and SECT images can be found in Figure $4 C$.

\section{Image quality parameters}

SNR and CNR values of VBI and VMI reconstructions can be found in Figure 5 and Appendix 2.

\section{VBIs}

SNR and CNR values of VBI reconstructions were inferior to the corresponding SECT images when taking the radiation exposure into account (see Figure 5). Non-linear regression suggests that a pair of source data with similar radiation exposure resulting in similar image quality cannot be found using the current reconstruction technique. Furthermore, the image quality of the $120 \mathrm{kVe}$ images was more dependent on the tube current at high $\mathrm{kVp}$, whereas the quality of the $100 \mathrm{kVe}$ images depended more on the low $\mathrm{kVp}$ dataset (see Table 1).

\section{VMIs}

Among the VMI reconstructions, $65 \mathrm{keV}$ images showed the highest SNR and CNR (see Appendix 2). Nonetheless, when taking the radiation exposure into account, all VMI reconstructions are inferior to all SECT images. However, non-linear regression suggests that $65 \mathrm{keV}$ reconstructions may reach similar SNR (but not $\mathrm{CNR}$ ) values as $135 \mathrm{kVp}$ images using the same radiation exposure (see Figure 5).

\section{Optimal scanning parameters}

The formulas of the graphs shown in Figure 5 were inserted into Eq. [1] and, thus, the minimum difference to the SECT images was calculated. The results can be found in Table 1 . In conclusion, using the optimal parameters, DECT could be performed with about $120 \%$ of the radiation exposure, however, resulting only in about $80 \%$ of the image quality of the corresponding SECT images.

\section{Discussion}

Our study used a 3D-printed phantom of the neck from 
100 kVe virtual blended images
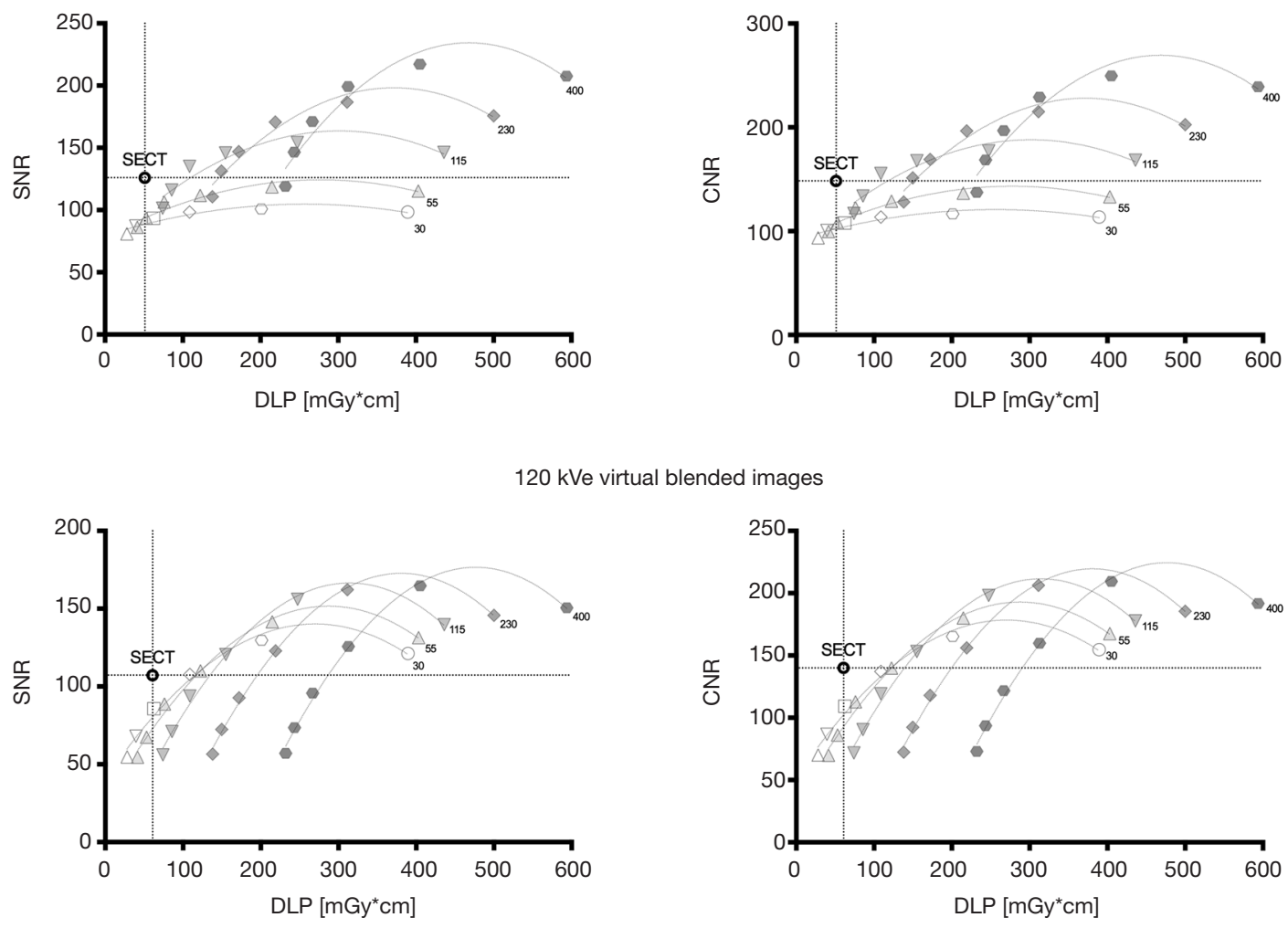

Virtual monochromatic images
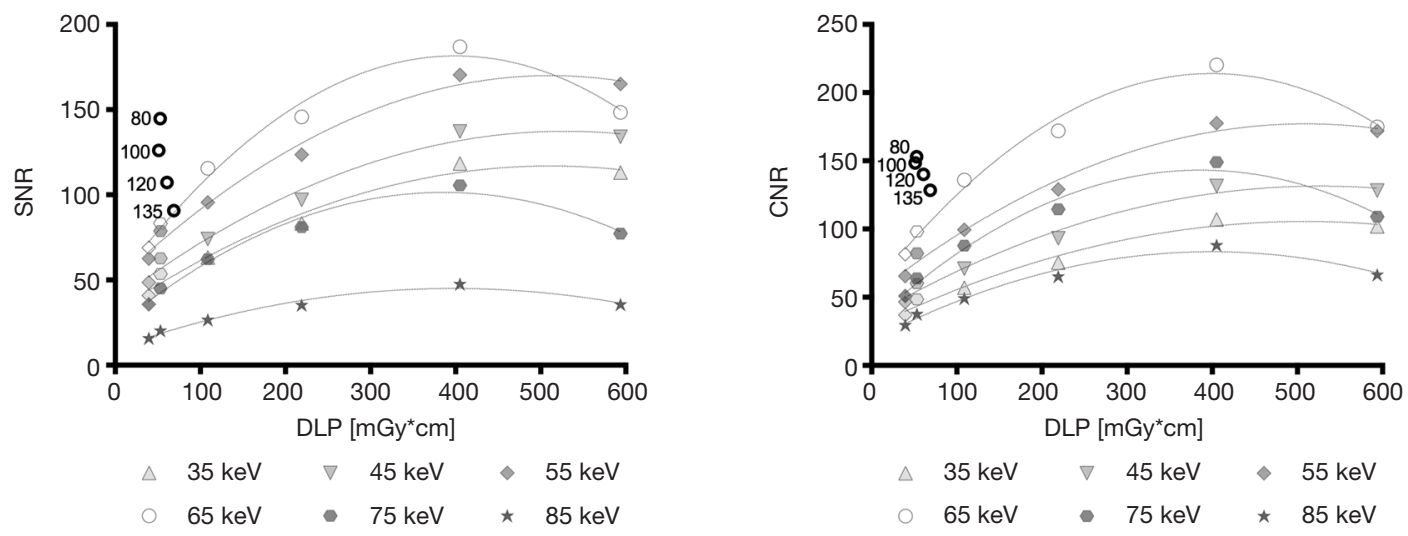

Figure 5 Comparison of image quality and radiation dose of dual-energy computed tomography (DECT) reconstructions to single-energy computed tomography (SECT) images that served as standard of reference. Signal-to-noise ratio (SNR) and contrast-to-noise ratio (CNR) values (y-axis) compared to the radiation exposure as measured by the dose length product (DLP; $\mathrm{x}$-axis) of virtual blended images in 100 and $120 \mathrm{kVe}$ reconstructions and selected virtual monochromatic images (35 to $85 \mathrm{keV}$ ) compared to SECT datasets. The left upper corner on the graph represents high image quality and low radiation exposure (thus, optimal balance) whereas the right lower corner represents low image quality and high exposure. The figure shows that neither actual measured DECT reconstructions nor extrapolations between the datapoints were able to achieve a similar or superior ratio of radiation exposure and image quality as SECT images (with the exception of $65 \mathrm{keV}$ VMI images that may meet the SNR/DLP of $135 \mathrm{kVp}$ SECT). 
Table 1 Optimal DECT protocol for VBI reconstructions with respect to image quality and radiation exposure

\begin{tabular}{|c|c|c|c|c|c|}
\hline Parameter & \multicolumn{5}{|c|}{ Source data combination } \\
\hline \multicolumn{6}{|l|}{ SNR } \\
\hline \multicolumn{6}{|l|}{100 kVe } \\
\hline Distance & 34.5 & 31.3 & 23.9 & 56.5 & 123.8 \\
\hline$\%$ SNR & \multicolumn{5}{|c|}{$83.0 \%(104.6 / 126)$} \\
\hline$\%$ DLP & \multicolumn{5}{|c|}{$120.5 \%(61.7 / 51.2)$} \\
\hline \multicolumn{6}{|l|}{$120 \mathrm{kVe}$} \\
\hline Distance & 23.7 & 28.6 & 44.3 & 87.4 & 155.5 \\
\hline \% DLP & $118.5 \%(72.1 / 60.8)$ & & & & \\
\hline \multicolumn{6}{|l|}{ CNR } \\
\hline \multicolumn{6}{|l|}{$100 \mathrm{kVe}$} \\
\hline Distance & 45.4 & 38.6 & 29.4 & 63.4 & 132.5 \\
\hline $\operatorname{DLP}(x)$ & 59.0 & 62.2 & 65.2 & 93.8 & 156.1 \\
\hline$\%$ CNR & \multicolumn{5}{|c|}{$82.3 \%(122.5 / 148.4)$} \\
\hline \% DLP & \multicolumn{5}{|c|}{$127.3 \%(65.2 / 51.2)$} \\
\hline \multicolumn{6}{|l|}{120 kVe } \\
\hline
\end{tabular}

SNR, signal to noise ratio; CNR, contrast to noise ratio. (x/y mAs): curve derived from DECT source data pairings with y mAs at $80 \mathrm{kVp}$; distance: distance on the graph (see Figure 5) of the corresponding SECT dataset to the DECT curve; DLP (x): $x$-value of the point on the curve nearest to the SECT point; \% SNR/CNR: ratio of the optimal DECT SNR to SECT, calculated SNR $\mathrm{DECT}_{\mathrm{SNR}} / \mathrm{SECT}_{\mathrm{S}}$, this equals $\mathrm{f}(\mathrm{x}) / \mathrm{SNR}$ SECT; $\%$ DLP: ratio of the optimal DECT DLP to SECT, calculated in the same manner.

actual patient data, thorough DECT scanning and reconstructions and comprehensive measurements to evaluate image quality and radiation dose of DECT in comparison to clinical standard SECT. We found that VBI reconstructions of 100 and $120 \mathrm{kVe}$ images showed a small but significant overestimation of $\mathrm{HU}$, when compared to corresponding SECT. HU of $75 \mathrm{keV}$ VMI corresponded well to $120 \mathrm{kVp}$ SECT, as found by other studies before (24). DECT showed a significant shortfall in image quality per radiation dose for all reconstructions (Figure 5). We found that under optimal tube current settings DECT reconstructions could achieve about $80 \%$ SNR and CNR by using $120 \%$ of the corresponding SECT radiation exposure. The optimal ratio of the tube current in high and low tube voltage DECT scans depended on the desired reconstruction: $120 \mathrm{kVe} \mathrm{VBI}$ images profited from rather high $\mathrm{mAs}$ in high $\mathrm{kVp}$ (e.g., 20/30 or 40/30 mAs), whereas $100 \mathrm{kVe}$ VBI profited from a higher tube current for low $\mathrm{kVp}$ data (e.g., $5 / 115$ or $10 / 55 \mathrm{mAs}$ ).

We conclude that the sequential DECT technique used for this study compared to clinical standard SECT goes along with either increased radiation exposure or 
with a decrease in image quality or a combination of both. However, we did not analyse a possible added value of the additional information that DECT is able to provide. Therefore, we cannot conclude whether the inferior image quality of DECT can be compensated in clinical practice. We propose that a sequential or dual-source DECT protocol should aim at approximately $120 \%$ of the radiation exposure of the standard SECT of the corresponding anatomic region; the ratio of tube currents in high and low tube voltage scans should be adapted to meet the desired main goal of reconstructions. However, this is not possible with all DECT machines: Fast-kVp-switching and duallayer detectors do not allow for an independent modulation of the tube current for low and high energy datasets.

Our results of Hounsfield units and image quality are in line with previous studies. Lam et al. found that the SNR and CNR values of VMI are optimal at $65 \mathrm{keV}(25)$. This is in line with our results that suggest that $65 \mathrm{keV}$ reconstructions might reach a similar ratio of radiation exposure and SNR as $135 \mathrm{kVp}$ SE images, but were not able to achieve similar CNR. This is most likely attributable to the fact that $65 \mathrm{keV}$ VMIs correspond to $90 \mathrm{kVp}$ SE in terms of $\mathrm{HU}$ (thus, being more sensitive iodinated contrast) but show higher noise compared to $135 \mathrm{kVp}$. However, they found that tumor visibility was improved using $40 \mathrm{keV}$ reconstructions because the contrast uptake is better visualized near the k-edge of iodine. Another phantom study achieved similar results on the image characteristics of VMI (26). Scholtz et al. also found a deviation of VBIs from corresponding SECT scans when using a standard linear blending algorithm (12). Some previous studies state that DECT image quality may be superior to corresponding SECT or may reduce the radiation exposure (27). The discrepancy to our findings might be attributable to differences in methodology, e.g., a study on actual patients with subjective image quality assessment compared to a phantom study with quantitative measurements, or in the DECT machine. Special techniques for DECT acquisition can possibly improve the image quality of secondary DECT reconstructions. For example, the tin filter technique achieves a better spectral separation and, therefore, might result in lower radiation exposure without compromising the image quality. The possible influence of the DECT reconstruction software on image quality might also explain differences to previous studies. Moreover, the physical properties of our phantom may have an influence. The attenuation of the phantom was mainly based on iodinated ink that has different dual-energy properties compared to the soft tissue or bone of a real patient. Therefore, the phantom does not fully reflect the effective $Z$ in a patient and measurements of DE values in the phantom will not compare to those in clinical practice. However, as one central advantage of DECT is its sensitivity for contrast medium, we believe that our results reflect the applicability.

Some limitations have to be discussed: We used DECT with sequential volume acquisition for our analysis as this is the preferred technique by the vendor. Therefore, our data cannot be directly applied to other DECT machines and protocols. However, the conclusions might in part be transferred to other sequential acquisition techniques and dual-source scanners. Spiral CT would result in higher radiation exposure (28). No beam hardening compensation was applied because the vendor does not allow the reconstruction of DECT raw-data sets with beam hardening compensation. Therefore, the results, especially for low keV VMIs might be impaired. In one of our DECT examinations we noticed an overexposure phenomenon of the detector during the high-energy scan that lead to a significant decrease of the image quality of the related secondary reconstructions. A multivariate analysis was not performed following the advice of our statistics team. We were not able to investigate the influence of different pairings on VMI images - neither the CT machine allowed for an independent adjustment of the tube current nor did the raw-data based software that was used for VMI reconstructions allow a separate selection of datasets. Furthermore, the details of the VMI reconstruction software remain hidden from the user. Future studies might go beyond those limitations, e.g., by using an independent software solution and a more sophisticated phantom approach that has more realistic dual-energy properties.

\section{Conclusions}

Based on the measurements on a 3D-printed anthropomorphic phantom of the neck, we were able to show that the sequential acquisition of DECT results in increased radiation exposure or decreased image quality when compared to SECT with respect to the usage of iodinated contrast and corresponding DECT applications. Optimal DECT protocols would apply an estimated $120 \%$ of the SECT radiation exposure and result in approximately $80 \%$ of the image quality. The tube current of the high and low energy dataset should be adapted to the desired DECT information, if the CT machine allows for a separate adjustment. Current VBI reconstructions overestimate the Hounsfield Units of different contrast concentrations 
leading to a demand of non-linear blending algorithms to achieve more accuracy.

\section{Acknowledgments}

Funding: None.

\section{Footnote}

Conflicts of Interest: All authors have completed the ICMJE uniform disclosure form (available at http://dx.doi. org/10.21037/qims-20-854). TD held talks for Canon Medical System. However, the company did not influence nor authorize the results of this study. MS reports grants from Bundesministerium für Wirtschaft und Energie (DE), during the conduct of the study; other from PhantomX $\mathrm{GmbH}$, outside the submitted work; in addition, MS has a patent DE202015104282 U1 issued, a patent US9924919B2 issued, a patent US10182786B2 issued, and a patent EP3135199A1 pending. BH reports grants to the Department of Radiology outside the submitted work from Abbott, Actelion Pharma, Bayer Schering Pharma, Bayer Vital, BRACCO Group, Bristol-Myers Squibb, Charité Research organisation GmbH, Deutsche Krebshilfe, Dt. Stiftung für Herzforschung, Essex Pharma, EU Programmes, Fibrex Medical Inc., Focused ultrasound Surgery Foundation, Fraunhofer Gesellschaft, Gurbet, INC Research, InSightec Ltd., IPSEN Pharma, Kendle/ MorphoSys AG, Lilly GmbH, Lundbeck GmbH, MeVis Medical Solutions AG, Nexus Oncology, Novartis, Parexel CRO Service, Perceptive, Pfizer GmbH, Philipps, sonofisaventis S.A., Siemens, Spectranetics GmbH, Terumo Medical Corporation, TNS Healthcare GmbH, Canon Medical, UCB Pharma, Wyeth Pharma, Zukunftsfond Berlin (TSB), outside the submitted work. Dr. PJ reports grants from Bundesministerium für Wirtschaft und Energie (DE), during the conduct of the study; other from PhantomX GmbH, outside the submitted work; in addition, PJ has a patent DE202015104282U1 issued, a patent US9924919B2 issued, a patent US10182786B2 issued, and a patent EP3135199A1 pending. The other author has no conflicts of interest to declare.

Ethical Statement: An approval of the institutional review board was waived for this study as no patients were involved.

Open Access Statement: This is an Open Access article distributed in accordance with the Creative Commons
Attribution-NonCommercial-NoDerivs 4.0 International License (CC BY-NC-ND 4.0), which permits the noncommercial replication and distribution of the article with the strict proviso that no changes or edits are made and the original work is properly cited (including links to both the formal publication through the relevant DOI and the license). See: https://creativecommons.org/licenses/by-nc-nd/4.0/.

\section{References}

1. Forghani R, De Man B, Gupta R. Dual-energy computed tomography: physical principles, approaches to scanning, usage, and implementation: Part 1. Neuroimaging Clin N Am 2017;27:371-84.

2. Forghani R, De Man B, Gupta R. Dual-energy computed tomography: physical principles, approaches to scanning, usage, and implementation: Part 2. Neuroimaging Clin N Am 2017;27:385-400.

3. Omoumi P, Becce F, Racine D, Ott JG, Andreisek G, Verdun FR. Dual-energy CT: basic principles, technical approaches, and applications in musculoskeletal imaging (Part 1). Semin Musculoskelet Radiol 2015;19:431-7.

4. Omoumi P, Verdun FR, Guggenberger R, Andreisek G, Becce F. Dual-energy CT: basic principles, technical approaches, and applications in musculoskeletal imaging (Part 2). Semin Musculoskelet Radiol 2015;19:438-45.

5. Lam S, Gupta R, Kelly H, Curtin HD, Forghani R. Multiparametric evaluation of head and neck squamous cell carcinoma using a single-source dual-energy CT with fast $\mathrm{kVp}$ switching: State of the Art. Cancers 2015;7:2201-16.

6. Forghani R, Roskies M, Liu X, Tan X, Mlynarek A, Payne RJ, Nair JR, Hier MP, Levental M. Dual-energy CT characteristics of parathyroid adenomas on 25 -and 55-second 4D-CT acquisitions: preliminary experience. J Comput Assist Tomogr 2016;40:806-14.

7. Fu F, He A, Zhang Y, Li B, Wan Y. Dua-energy virtual noncontrast imaging in diagnosis of cervical metastasis lymph nodes. J Cancer Res Ther 2015;11 Suppl 2:C202-4

8. Jiang XY, Zhang SH, Xie QZ, Yin ZJ, Liu QY, Zhao MD, Li XL, Mao XJ. Evaluation of virtual noncontrast images obtained from dual-energy CTA for diagnosing subarachnoid hemorrhage. AJNR Am J Neuroradiol 2015;36:855-60.

9. Kuno H, Sakamaki K, Fujii S, Sekiya K, Otani K, Hayashi R, Yamanaka T, Sakai O, Kusumoto M. Comparison of MR imaging and dual-energy CT for the evaluation 
of cartilage invasion by laryngeal and hypopharyngeal squamous cell carcinoma. AJNR Am J Neuroradiol 2018;39:524-31.

10. Ma G, Yu Y, Duan H, Dou Y, Jia Y, Zhang X, Yang C, Chen X, Han D, Guo C, He T. Subtraction CT angiography in head and neck with low radiation and contrast dose dual-energy spectral CT using rapid kVswitching technique. Br J Radiol 2018;91:20170631.

11. Bahig H, Lapointe A, Bedwani S, de Guise J, Lambert L, Filion E, Roberge D, Letourneau-Guillon L, Blais D, Ng SP, Nguyen-Tan PF. Dual-energy computed tomography for prediction of loco-regional recurrence after radiotherapy in larynx and hypopharynx squamous cell carcinoma. Eur J Radiol 2019;110:1-6.

12. Scholtz JE, Husers K, Kaup M, Albrecht M, Schulz B, Frellesen C, Bodelle B, Wagenblast J, Kerl JM, Bauer RW, Lehnert T, Vogl TJ, Wichmann JL. Nonlinear image blending improves visualization of head and neck primary squamous cell carcinoma compared to linear blending in dual-energy CT. Clin Radiol 2015;70:168-75.

13. Neuhaus V, Grosse Hokamp N, Abdullayev N, Maus V, Kabbasch C, Mpotsaris A, Maintz D, Borggrefe J. Comparison of virtual monoenergetic and polyenergetic images reconstructed from dual-layer detector CT angiography of the head and neck. Eur Radiol 2018;28:1102-10.

14. Forghani R, Kelly H, Yu E, Belair M, Letourneau-Guillon L, Le H, Proulx F, Ong T, Tan X, Curtin HD, Levental M. Low-Energy Virtual Monochromatic Dual-Energy Computed Tomography Images for the Evaluation of Head and Neck Squamous Cell Carcinoma: A Study of Tumor Visibility Compared With Single-Energy Computed Tomography and User Acceptance. J Comput Assist Tomogr 2017;41:565-71.

15. Chen Y, Xue H, Jin ZY, Zhang J, Sun H, Wang X, Zhang ZH, Zhang DM, Lu GM, Zhang ZQ, Schoepf UJ, Bucher AM, Wolla CD, Wang Y. 128-slice acceletated-pitch dual energy CT angiography of the head and neck: comparison of different low contrast medium volumes. PLoS One 2013;8:e80939.

16. Große Hokamp N, Laukamp KR, Lennartz S, Zopfs D, Abdullayev N, Neuhaus VF, Maintz D, Borggrefe J. Artifact reduction from dental implants using virtual monoenergetic reconstructions from novel spectral detector CT. Eur J Radiol 2018;104:136-42.

17. Liao E, Srinivasan A. Applications of dual-energy computed tomography for artifact reduction in the head, neck, and spine. Neuroimaging Clin N Am 2017;27:489-97.

18. Nair JR, DeBlois F, Ong T, Devic S, Tomic N, Bekerat H, Rosenbloom L, Sultanem K, Forghani R. Dual-Energy CT: Balance Between Iodine Attenuation and Artifact Reduction for the Evaluation of Head and Neck Cancer. J Comput Assist Tomogr 2017;41:931-6.

19. May MS, Bruegel J, Brand M, Wiesmueller M, Krauss B, Allmendinger T, Uder M, Wuest W. Computed Tomography of the Head and Neck Region for Tumor Staging-Comparison of Dual-Source, Dual-Energy and Low-Kilovolt, Single-Energy Acquisitions. Invest Radiol 2017;52:522-8.

20. Paul J, Mbalisike EC, Nour-Eldin NE, Vogl TJ. Dualsource 128-slice MDCT neck: radiation dose and image quality estimation of three different protocols. Eur J Radiol 2013;82:787-96.

21. Tawfik AM, Kerl JM, Razek AA, Bauer RW, Nour-Eldin NE, Vogl TJ, Mack MG. Image quality and radiation dose of dual-energy CT of the head and neck compared with a standard 120-kVp acquisition. AJNR Am J Neuroradiol 2011;32:1994-9.

22. Jahnke P, Limberg FR, Gerbl A, Ardila Pardo GL, Braun VP, Hamm B, Scheel M. Radiopaque Three-dimensional Printing: A Method to Create Realistic CT Phantoms. Radiology 2017;282:569-75.

23. Jahnke P, Schwarz S, Ziegert M, Schwarz FB, Hamm B, Scheel M. Paper-based 3D printing of anthropomorphic CT phantoms: feasibility of two construction techniques. Eur Radiol 2019;29:1384-90.

24. Jacobsen MC, Schellingerhout D, Wood CA, Tamm EP, Godoy MC, Sun J, Cody DD. Intermanufacturer Comparison of Dual-Energy CT Iodine Quantification and Monochromatic Attenuation: A Phantom Study. Radiology 2018;287:224-34.

25. Lam S, Gupta R, Levental M, Yu E, Curtin HD, Forghani R. Optimal Virtual Monochromatic Images for Evaluation of Normal Tissues and Head and Neck Cancer Using Dual-Energy CT. AJNR Am J Neuroradiol 2015;36:1518-24.

26. Sakabe D, Funama Y, Taguchi K, Nakaura T, Utsunomiya D, Oda S, Kidoh M, Nagayama Y, Yamashita Y. Image quality characteristics for virtual monoenergetic images using dual-layer spectral detector CT: Comparison with conventional tube-voltage images. Phys Med 2018;49:5-10.

27. Suntharalingam S, Stenzel E, Wetter A, Guberina N, Umutlu L, Schlosser T, Nassenstein K. Third generation dual-energy CT with 80/150 Sn $\mathrm{kV}$ for head and neck 
tumor imaging. Acta Radiol 2019;60:586-92.

28. Kang EJ, Lee KN, Kim DW, Kim BS, Choi S, Park BH, Oh JY. Triple rule-out acute chest pain evaluation using a 320-row-detector volume CT: a comparison of the widevolume and helical modes. Int J Cardiovasc Imaging 2012;28 Suppl 1:7-13.

Cite this article as: Diekhoff T, Scheel M, Kress W, Hamm B, Jahnke P. Dual-energy computed tomography of the neck-optimizing tube current settings and radiation dose using a 3D-printed patient phantom. Quant Imaging Med Surg 2021;11(4):1144-1155. doi: 10.21037/qims-20-854 


\section{Appendix 1}

Appendix 1A Dunnett's multiple comparison test of the mean HU of $80 \mathrm{kVp}$ single energy energy CT (SECT) and the $80 \mathrm{kVe}$ virtual blended images (VBI) reconstructed from different dual energy CT (DECT) parings (high $\mathrm{kVp} /$ low $\mathrm{kVp}$ )

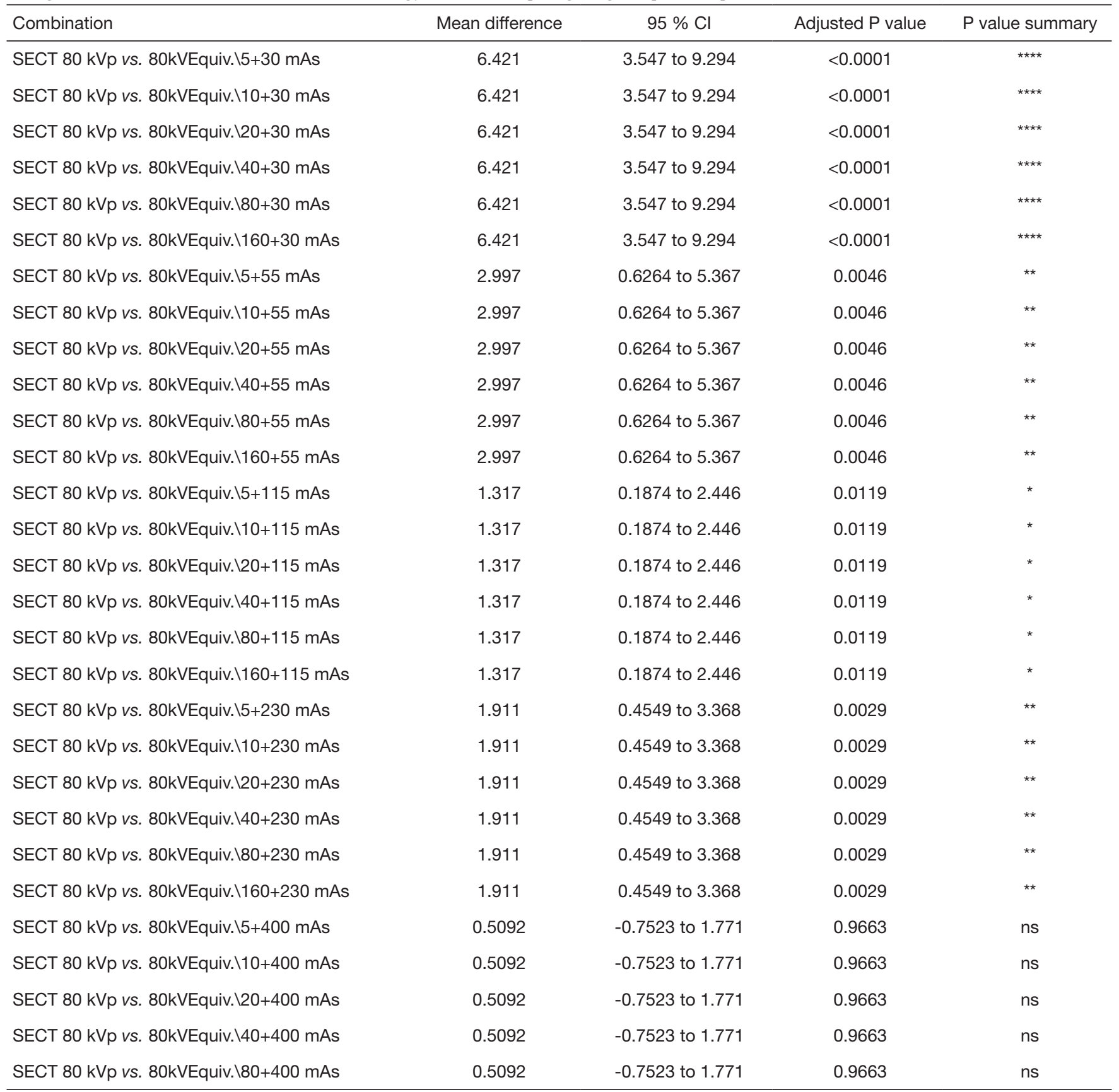

ns, $\mathrm{P}>0.05 ;{ }^{*}, \mathrm{P}=0.05-0.01 ;{ }^{* \star}, \mathrm{P}=0.01-0.001 ;{ }^{* \star \star *}, \mathrm{P}<0.0001$. 
Appendix 1B Dunnett's multiple comparison test of the mean HU of $100 \mathrm{kVp}$ single energy energy CT (SECT) and the $100 \mathrm{kVe}$ virtual blended images (VBI) reconstructed from different dual energy CT (DECT) parings (high $\mathrm{kVp} /$ low $\mathrm{kVp}$ )

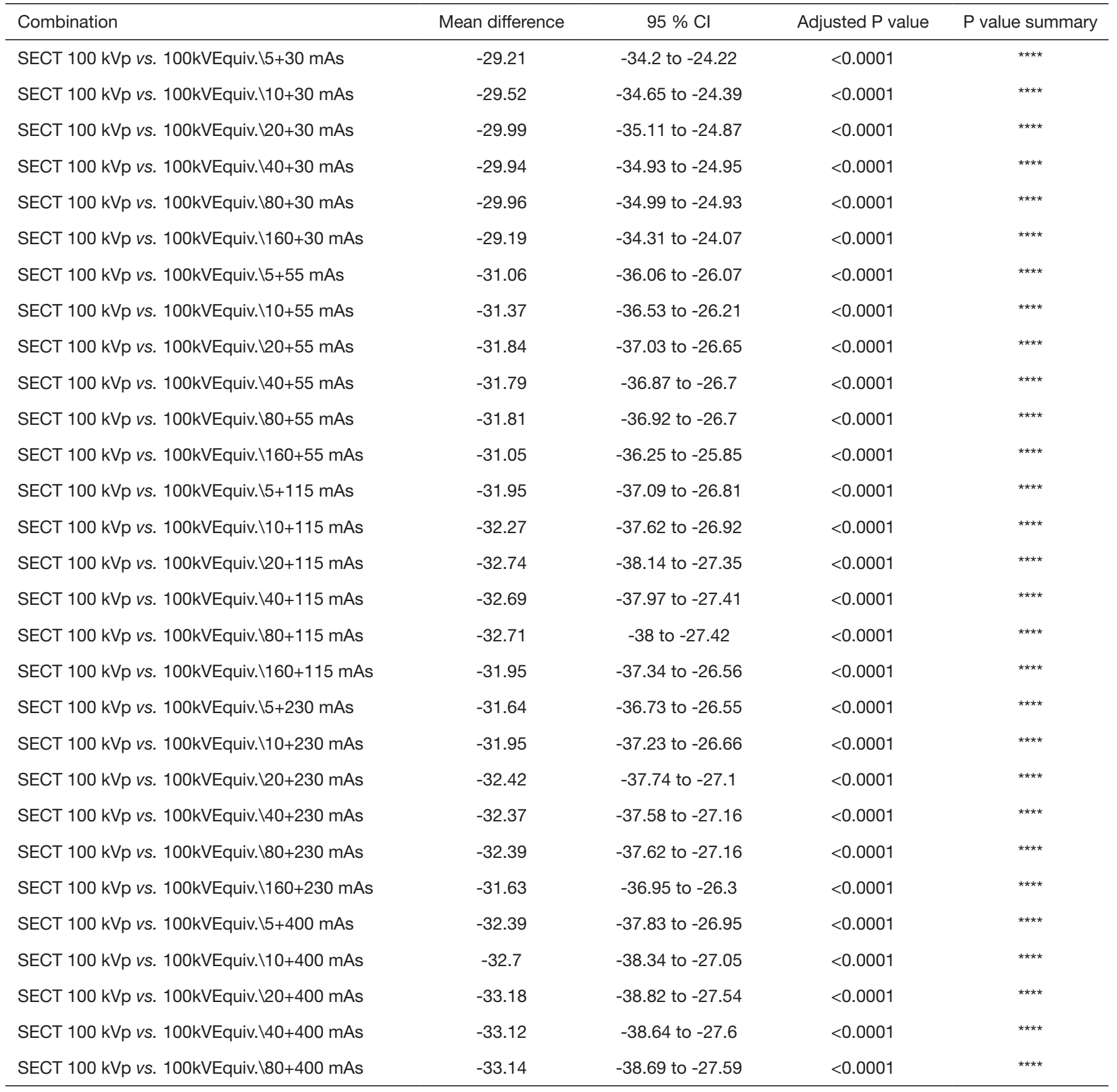
****, $\mathrm{P}<0.0001$. 
Appendix 1C Dunnett's multiple comparison test of the mean HU of $120 \mathrm{kVp}$ single energy energy CT (SECT) and the $120 \mathrm{kVe}$ virtual blended images (VBI) reconstructed from different dual energy CT (DECT) parings (high $\mathrm{kVp} /$ low $\mathrm{kVp}$ )

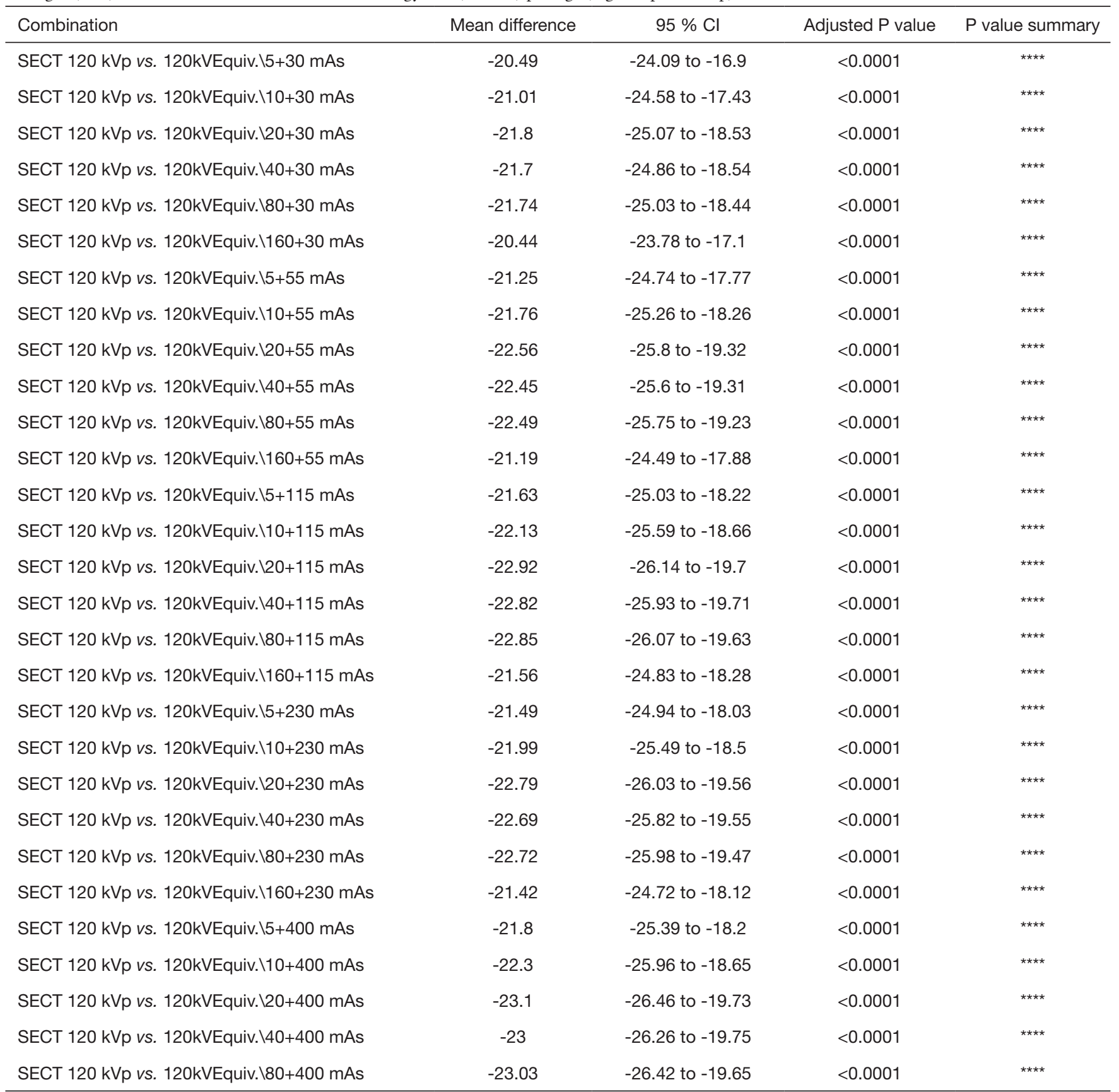
****, $\mathrm{P}<0.0001$. 
Appendix 1D Dunnett's multiple comparison test of the mean HU of the $135 \mathrm{kVp}$ single energy energy CT (SECT) and the $135 \mathrm{kVe}$ virtual blended images (VBI) reconstructed from different dual energy CT (DECT) parings (high $\mathrm{kVp} /$ low $\mathrm{kVp}$ )

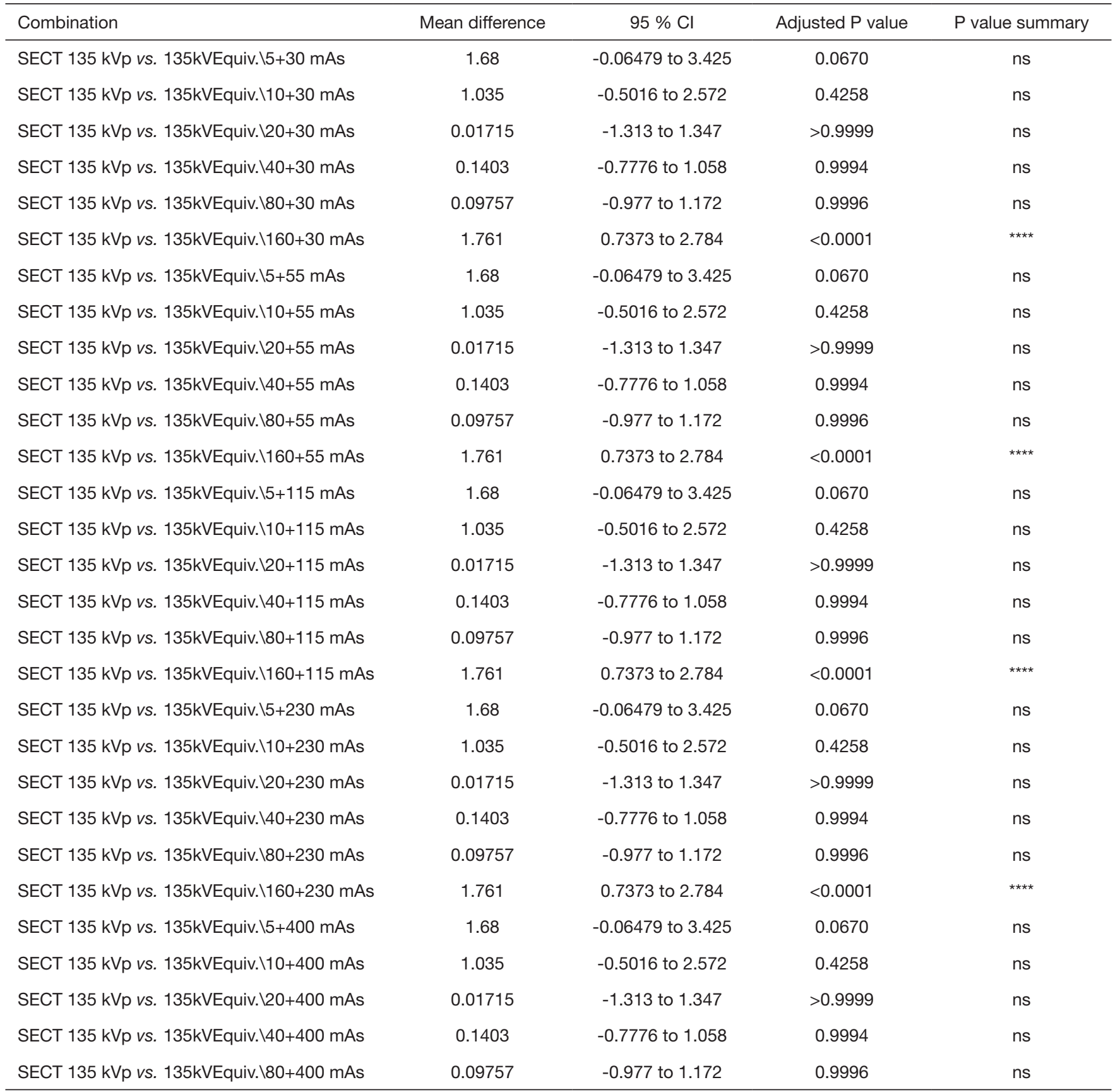

ns, $\mathrm{P}>0.05 ;{ }^{* * \star *}, \mathrm{P}<0.0001$. 


\section{Appendix 2}
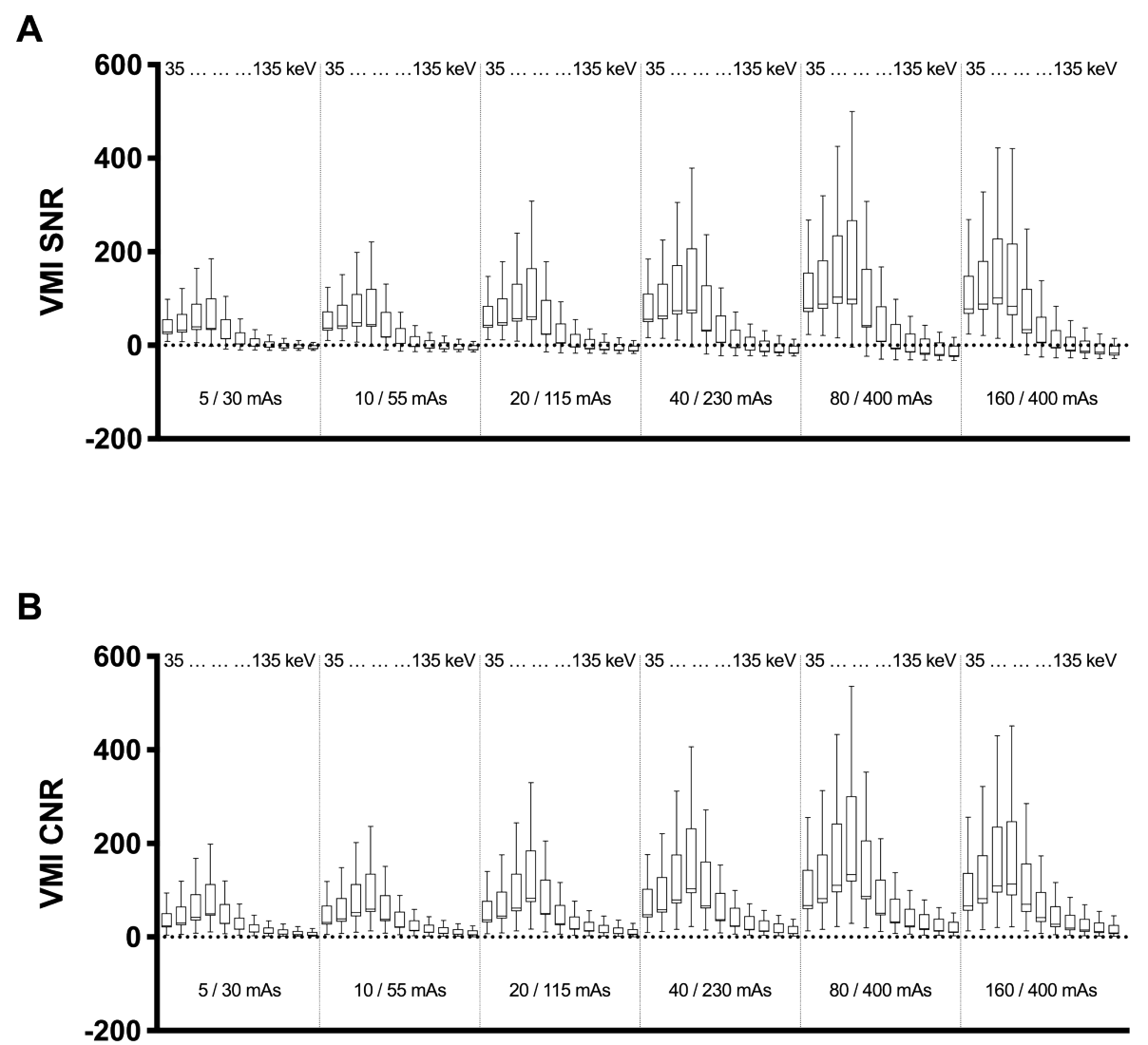

Appendix 2 Signal to noise ratio (SNR; A) and contrast to noise ratio (CNR; B) of the virtual monochromatic images (VMI) reconstructed from dual energy computed tomography (DECT). A and B: For each radiation dose the SNR and CNR values are highest for $65 \mathrm{keV}$ reconstructions. 